\title{
Successful management of aortoesophageal fistula by combining endoscopic cyanoacrylate injection and endovascular stent grafting
}
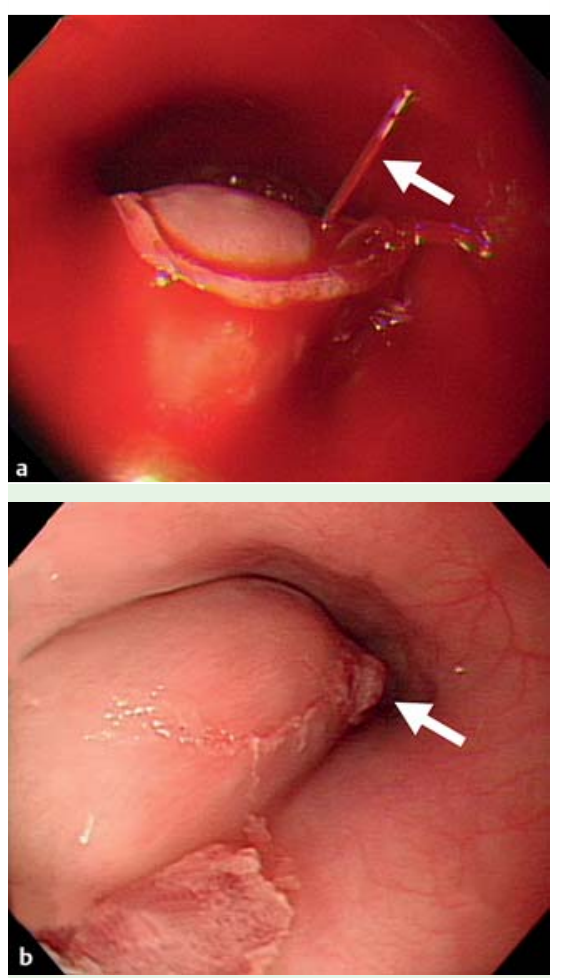

Fig. 1 a Esophagogastroduodenoscopy (EGD) showing one 3-cm mass lesion with spurting bleeding $26 \mathrm{~cm}$ from incisors (arrow). b EGD after endovascular stent grafting, showing a submucosal mass measuring $3 \mathrm{~cm} \times 1.8 \mathrm{~cm}$ with an erosive tip and $\mathrm{N}$-butyl-2-cyanoacrylate coating (arrow).

Aortoesophageal fistula (AEF) is a rare cause of catastrophic gastrointestinal bleeding. Thoracic aortic aneurysms are the leading cause of AEF [1]. Early diagnosis and prompt endovascular and/or surgical intervention before massive exsanguinating hemorrhage are key to survival [2]. Temporary endoscopic management with esophageal stent placement has been reported as a bridge therapy $[3,4]$. We describe a case of AEF with active bleeding noted during an endoscopic examination that was treated with injection of $\mathrm{N}$-butyl-2-cyanoacrylate (NBCA, Histoacryl), followed by successful placement of an endovascular aortic stent graft.

A 69-year-old man with a history of ruptured diverticula that had been managed surgically presented to the emergency department with a 1-day history of chest pain and tarry stool, with hema-

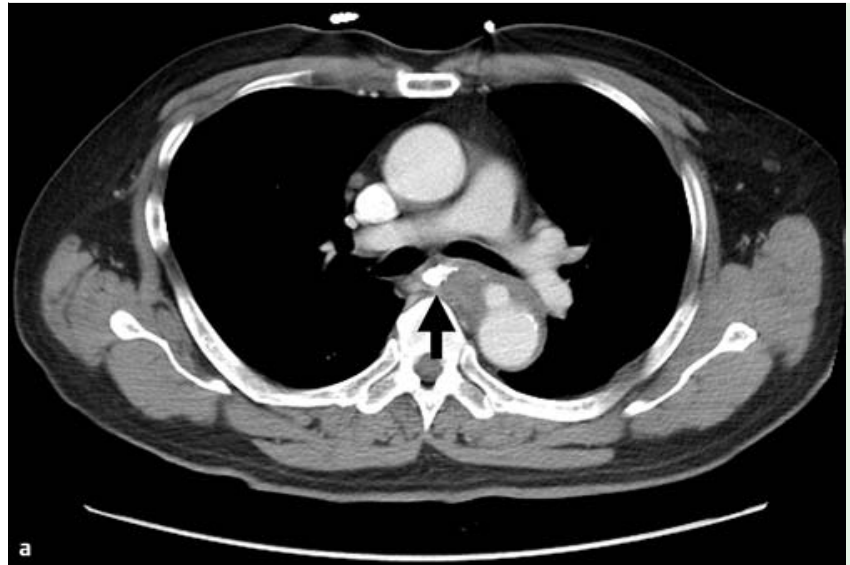

Fig. 2 a Chest CT showing a 5-mm saccular aneurysm on the anterior wall of the mid descending thoracic aorta, consistent with aortic-esophageal fistula. A high-attenuation lesion in the mid esophagus abutting the aortic aneurysm is consistent with a deposition of $\mathrm{N}$-butyl-2-cyanoacrylate with lipiodol (arrow). b Three-dimensional volume-rendered CT

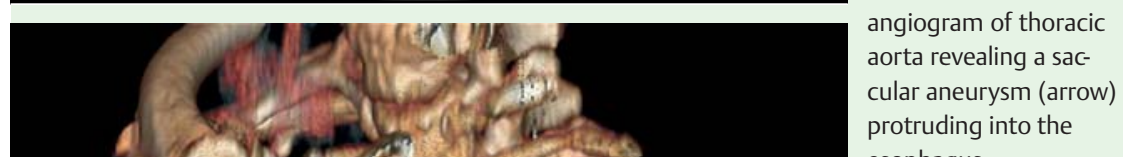
protruding into the esophagus. temesis and hematochezia on the morning of admission. His heart rate was 95 beats/min and his blood pressure was 214/111 mmHg. Laboratory data showed a rapid decline in hemoglobin $(13.1 \mathrm{~g} / \mathrm{dL}$ to $10.2 \mathrm{~g} / \mathrm{dL}$ in 3 hours). Emergent esophagogastroduodenoscopy (EGD) revealed a $3-\mathrm{cm}$ submucosal mass lesion with a spurting vessel $26 \mathrm{~cm}$ from the incisors ( Fig. 1 a).

Two milliliters of NBCA mixed with lipiodol was injected locally, achieving temporary hemostasis. Contrast-enhanced computed tomography of the chest disclosed a descending thoracic aortic aneurysm with a 5-mm AEF ( Fig. 2).

Aorta angiography revealed a 5-mm aneurysm without active extravasation in the mid thoracic aorta ( $\mathbf{F i g} . \mathbf{3 a}$ ).

The thoracic aortic aneurysm was repaired with a Cook Zenith II (34 mm× $77 \mathrm{~mm}$ ) stent graft without complications ( Fig. 3b). EGD after stent grafting the same day showed a submucosal mass measuring $3 \mathrm{~cm} \times 1.8 \mathrm{~cm}$ with an erosive lesion and NBCA coating ( $\bullet$ Fig. $1 \mathbf{b}$ ). 

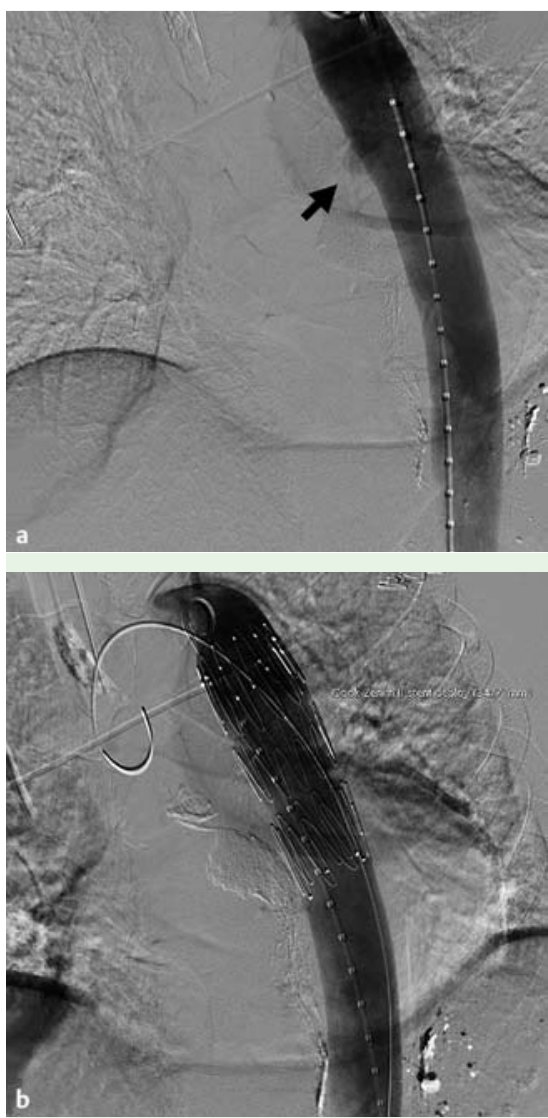

Fig. 3 a Aortogram showing a 5-mm aneurysm (arrow) from the mid thoracic aorta just below the carina without active extravasation into the esophagus. b After stent grafting, the aneurysm has disappeared.
After stent grafting, the patient suffered from intermittent episodes of chest and back pain. Intravenous broad-spectrum antibiotics were administered for a total of 8 weeks, followed by oral antibiotics. The patient recovered and remained healthy at 8 months follow-up.

Endoscopy_UCTN_Code_CCL_1AB_2AC_3AG

\section{Competing interests: None}

\section{K.-C. Tseng ${ }^{1,2}$, C.-W. Lin ${ }^{3}$, J. W.-H. Tan}

${ }^{1}$ Department of Internal Medicine, Buddhist Dalin Tzu Chi General Hospital, Chia-Yi, Taiwan

2 School of Medicine, Tzuchi University, Hualien, Taiwan

3 Department of Radiology, Buddhist Dalin Tzu Chi General Hospital, Chia-Yi, Taiwan

4 Department of Cardiovascular Surgery, Buddhist Dalin Tzu Chi General Hospital, Chia-Yi, Taiwan

\section{References}

1 Léobon B, Roux D, Mugniot A et al. Endovascular treatment of thoracic aortic fistulas. Ann Thorac Surg 2002; 74: 247-249

2 Topel I, Stehr A, Steinbauer MG et al. Surgical strategy in aortoesophageal fistulae: endovascular stentgrafts and in situ repair of the aorta with cryopreserved homografts. Ann Surg 2007; 246: 853-859

3 Park DH, Park JH, Lee SH et al. Temporary placement of a covered metal stent for the management of a bleeding aortoesophageal fistula. Endoscopy 2007; 39 (Suppl 1): E61 E62

4 Zuber-Jerger I, Hempel U, Rockmann F et al. Temporary stent placement in 2 cases of aortoesophageal fistula. Gastrointest Endosc 2008; 68: 599-602

\section{Bibliography}

DOI $10.1055 / \mathrm{s}-0030-1256167$

Endoscopy 2011; 43: E135-E136

(c) Georg Thieme Verlag KG Stuttgart · New York . ISSN 0013-726X

\section{Corresponding author}

\section{J. W.-H. Tan, MD}

Department of Cardiovascular Surgery Buddhist Dalin Tzu Chi General Hospital No. 2, Min-Sheng Road Dalin Town

Chia-Yi

Taiwan, 622

Fax: +886-5-2648006

neugine@gmail.com 